\section{Pacientes Estandarizados en la Formación de Habilidades Clínicas en Educación Médica de Pregrado: Réplica}

\section{Standardized Patients in Clinical Skills Training in Undergraduate Medical Education: Reply}

\section{Sr. Editor:}

Hemos leído con mucho interés la carta de Solís et al, en relación a nuestro Artículo de Revisión ${ }^{1}$. Agradecemos los comentarios y el aporte en relación al uso de pacientes estandarizados en la educación médica de pregrado.

Desde nuestro punto de vista, el desarrollo de habilidades comunicacionales es parte esencial de la educación en Medicina. Reflejo de esto es el lugar que ocupa, por ejemplo, en el currículo de pregrado y postgrado de Medicina de la Pontificia Universidad Católica de Chile ${ }^{2}$. Esta experiencia se ha traducido también en diversos estudios y publicaciones $^{3-5}$.

El uso de la simulación de pacientes estandarizados para entrenamiento de encuentros clínicos y habilidades comunicacionales, se extiende por más de 4 décadas 6 . Tal como se describió en nuestro artículo, las clasificaciones existentes de los tipos de simulación, ampliamente difundidas y aceptadas, coinciden en considerar a los pacientes estandarizados como una variante fundamental ${ }^{7-10}$. En dichas clasificaciones, como la de Ziv (2003), coexisten herramientas o aproximaciones a la simulación de alta y baja fidelidad, con o sin tecnología involucrada.

En ese sentido, queremos nuevamente enfatizar que "Simulación" se define como una técnica, no una tecnología, para sustituir o ampliar las experiencias reales a través de experiencias guiadas, que evocan o replican aspectos sustanciales del mundo real, de una forma totalmente interactiva ${ }^{11}$. Como tal, el concepto no es privativo de los "Simuladores" que mencionan Solís et al en su carta, sino que considera todas aquellas herramientas que permiten alcanzar este objetivo (tecnologías de realidad aumentada, simulaciones computacionales o escenarios con pacientes estandarizados).

Mención aparte merece el insistir en un aspecto que ya mencionamos en nuestra revisión, respecto al concepto de "fidelidad". Este término clásicamente se ha utilizado para definir el grado de realismo de una experiencia simulada. Sin embargo, en algunas oportunidades se ha utilizado erróneamente para definir complejidad o tecnología: la fidelidad no es siempre proporcional a la complejidad y a la tecnología utilizada. A modo de ejemplo, cuando se realiza la entrevista médica a un paciente simulado, al que además se le realizará una otoscopia, se trata de una simulación de muy alta fidelidad ya que se acerca mucho a la realidad; sin embargo, es un escenario de baja complejidad y escasa tecnología.

En este contexto y con estas dos definiciones en mente, queremos reiterar que la fidelidad de simulación claramente no estaría definida por el nivel tecnológico involucrado, sino por sus características intrínsecas y el nivel de realismo que involucran.

Un ejemplo de ello puede ser la simulación híbrida, que basa su utilidad en el hecho de que los pacientes estandarizados proporcionan un nivel de realismo imposible de alcanzar sólo con maniquíes. De esta forma, al mezclar un paciente entrenado (alta fidelidad, baja complejidad) con un simulador de tarea parcial (baja fidelidad, baja complejidad), como por ejemplo ponerle un brazo de punción adosado a su cuerpo, permite a los estudiantes la posibilidad de realizar procedimientos invasivos, como la instalación de una vía venosa, situación que no sería posible con los pacientes estandarizados solos. Cuando se combinan ambas técnicas, los estudiantes entrenan habilidades de comunicación y habilidades motoras, en una sola simulación (alta complejidad y alta fidelidad del escenario, a expensas de escasa tecnología involucrada $)^{12}$.

Finalmente, el interés suscitado por nuestro artículo refuerza el valor que esta herramienta (en todas sus formas) tiene para la formación de profesionales y técnicos del área de la Salud y enriquecer el conocimiento de esta disciplina en nuestro país.

\section{Marcia Corvetto ${ }^{1}$, Fernando Altermatt ${ }^{2}$ ${ }^{1,2}$ División de Anestesiología, Escuela de Medicina, Pontificia Universidad Católica de Chile. Santiago de Chile.}




\section{Referencias}

1. Corvetto M, Bravo MP, Montana R, Utili F, Escudero E, Boza C, et al. [Simulation in medical education: a synopsis]. Rev Med Chile 2013; 141 (1): 70-9.

2. Moore P, Gómez G, Kurtz S. Doctor-patient communication: one of the basic competencies, but different. Atencion primaria/Sociedad Espanola de Medicina de Familia y Comunitaria. 2012; 44 (6): 358-65.

3. Gómez G, Moore P, Araos-Baeriswyl E. [Learning doctor-patient communication: does co-teaching help?] Rev Med Chile 2012; 140 (3): 396-403.

4. Moore P, Vargas A, Núñez S, Macchiavello S. [A study of hospital complaints and the role of the doctor-patient communication]. Rev Med Chile 2011; 139 (7): 880-5.

5. Moore P, Gómez G, Kurtz S, Vargas A. [Doctor patient communication: which skills are effective?] Rev Med Chile 2010; 138 (8): 1047-54.

6. Levine AI, Swartz MH. Standardized patients: the "other" simulation. J Crit Care 2008; 23 (2): 179-84.
7. Ziv A, Wolpe PR, Small SD, Glick S. Simulation-based medical education: an ethical imperative. Acad Med 2003; 78 (8): 783-8.

8. Lane J, Slavin S, Ziv A. Simulation in medical education: A review. Simulation \& Gaming 2001; 32 (3): 297-314.

9. Maran NJ, Glavin RJ. Low - to high-fidelity simulation-a continuum of medical education? Med Educ 2003; 37 Suppl 1: 22-8.

10. Alinier G. A typology of educationally focused medical simulation tools. Med Teach 2007; 29 (8): e243-50.

11. Gaba DM. The future vision of simulation in health care. Qual Saf Health Care 2004; 13 Suppl 1: i2-10.

12. http://cgea.net/CGEApstryudkowsky.pdf.

Correspondencia a:

Dra. Marcia Corvetto

Marcoleta 367, tercer piso

División de Anestesiología. Pontificia Universidad Católica de Chile, Santiago, Chile.

Teléfono: 562-3543270. Fax 562-3543270

E-mail: mcorvett@med.puc.cl 